\title{
Clinical pharmacokinetic interactions between antiepileptic drugs and hormonal contraceptives
}

\author{
Doodipala Samba Reddy, PhD, RPh [Associate Professor] \\ Department of Neuroscience and Experimental Therapeutics, College of Medicine, Texas A\&M \\ Health Science Center, 228 Reynolds Medical Building, College Station, TX 77843, USA, Tel.: +1 \\ 979862 2852, Fax: +1 979845 0790, reddy@medicine.tamhsc.edu
}

\begin{abstract}
Contraceptive management in women with epilepsy is critical owing to the potential maternal and fetal risks if contraception or seizure management fails. This article briefly describes the pharmacokinetic interactions between antiepileptic drugs (AEDs) and hormonal contraceptives and the rational strategies that may overcome these risks. Hormonal contraception, including the use of oral contraceptives (OCs), is widely used in many women with epilepsy - there is no strong evidence of seizures worsening with their use. AEDs are the mainstay for seizure control in women with epilepsy. However, there are many factors to consider in the choice of AED therapy and hormonal contraception, since some AEDs can reduce the efficacy of OCs owing to pharmacokinetic interactions. Estrogens and progestogens are metabolized by cytochrome P450 3A4. AEDs, such as phenytoin, phenobarbital, carbamazepine, felbamate, topiramate, oxcarbazepine and primidone, induce cytochrome P450 3A4, leading to enhanced metabolism of either or both the estrogenic and progestogenic component of OCs, thereby reducing their efficacy in preventing pregnancy. OCs can also decrease the concentrations of AEDs such as lamotrigine and, thereby, increase the risk of seizures. Increased awareness of AED interactions may help optimize seizure therapy in women with epilepsy.
\end{abstract}

\section{Keywords}

epilepsy; estrogen; oral contraceptive; pregnancy; progesterone

\begin{abstract}
Epilepsy affects an estimated 25 million women worldwide. There are approximately 1.5 million women of childbearing age with epilepsy in the USA [1,2], and epilepsy is associated with special concerns for many of these women [2,3]. There is abundant evidence of hormone effects on seizures, since many women report that their seizure patterns change at puberty, at different stages of the menstrual cycle and with the menopause. The natural fluctuation in steroid hormones that occurs during the menstrual cycle significantly influence seizure patterns and potentially influence drug therapy [4,5]. An estimated 10 million women in the USA and 100 million women worldwide use hormonal contraceptives [6]. Women using contraception must be aware of potential interactions between hormone contraceptives and antiepileptic drugs (AEDs) in order to ensure adequate efficacy and safety of both classes of drugs. However,
\end{abstract}

\footnotetext{
(C) 2010 Expert Reviews Ltd

Financial \& competing interests disclosure

The author's research program was funded by the NIH grants NS051398 and NS052158. The author has no other relevant affiliations or financial involvement with any organization or entity with a financial interest in or financial conflict with the subject matter or materials discussed in the manuscript apart from those disclosed.

No writing assistance was utilized in the production of this manuscript.
} 
many women are unaware of risks associated with drug interactions of hormonal contraceptives $[7,8]$.

This article describes the interactions between steroid hormones and AEDs in order to understand the implications for effective contraception and seizure management in women with epilepsy.

\section{Influence of steroid hormones on seizures}

Understanding the impact of steroid hormones on seizures is essential to determine the choice of hormonal contraception prescribed for women with epilepsy. Many women with epilepsy may experience seizures in a predictable fashion around menstrual periods. Women can experience an increase in seizure activity at the time of ovulation (when estrogen levels rise) and at the start of menstruation (when progesterone levels fall). The rhythmic occurrence of seizures in relation to the menstrual cycle is referred to as catamenial epilepsy, a common hormone-sensitive seizure disorder. Catamenial epilepsy is characterized by seizures that cluster around specific points in the menstrual cycle with three common patterns: perimenstrual, periovulatory and inadequate luteal phase [9]. Recent studies indicate that catamenial epilepsy affects $39-60 \%$ of the women with epilepsy [10,11]. In normal cycles, the number of seizures during perimenstrual and follicular phases is counted, and a twofold or greater increase in frequency can be used as criteria for the diagnosis of catamenial epilepsy (see [5]). Overall, one in three women with epilepsy show catamenial seizure exacerbation, more often with the perimenstrual pattern.

Cyclical changes in the circulating levels of estrogens and progesterone during the menstrual cycle play a key role in seizure susceptibility in women with epilepsy (see [5]). Generally, estrogens are found to be proconvulsant, while progesterone has powerful antiseizure effects and reduces seizure occurrence. Estradiol has been shown to have significant proconvulsant effects and increases neuronal excitability [5]. Progesterone is an anticonvulsant hormone $[12,13]$. Women with epilepsy are prone to seizures in response to decreased levels of progesterone during perimenstrual periods [14]. Consequently, periovulatory seizure exacerbation has been attributed to the midcycle surge of estrogen that is relatively unopposed by progesterone until the early luteal phase. The protective effects of progesterone are found to be due to its metabolic conversion in the brain to allopregnanolone ( $3 \alpha$-hydroxy-5 $\alpha$ pregnan-20-one), a neurosteroid and potent positive modulator of $\mathrm{GABA}_{\mathrm{A}}$ receptors [13]. In addition, there is emerging evidence that endogenous neurosteroids, including those derived from adrenal steroid hormones and circulating androgens, substantially influence the development or occurrence of seizures [5,14].

Endocrine dysfunction is common in women with epilepsy. Seizures contribute to abnormal menstrual cycles and reduced reproduction, probably as a result of the impact of seizures on the hypothalamic-pituitary axis, which controls gonadal hormone production. Consequently, women with epilepsy have a significantly higher possibility of developing disorders such as polycystic ovaries [15,16]. An estimated $30 \%$ of women with epilepsy experience irregular menstrual cycles, including lack of menstruation or lengthy or short menstrual cycles. Some AEDs alter the hypothalamic-pituitary axis function directly or by feedback regulation through gonodal steroid hormones [17]. Increase in serum sex hormone-binding globulin (SHBG) levels in women with epilepsy using AEDs lead to diminished estradiol bioactivity, which may result in reduced fertility $[18,19]$.

\section{AEDs for women with epilepsy}

The use of AEDs are the mainstay for the management of seizures in women with epilepsy. Currently, AEDs approved by the US FDA are generally classified as one of two generations 
(Table 1). The first-generation (standard) agents include carbamazepine, phenobarbital, phenytoin, primidone, ethosuximide and valproic acid. The second-generation (newer) agents include topiramate, felbamate, lamotrigine, tiagabine, gabapentin, levetiracetam, zonisamide and pregabalin. Many AEDs act by inhibition of voltage-gated sodium and calcium channels, or by potentiation of GABAergic inhibition [20,21]. Clinical experience suggests that the newer AEDs have a broader spectrum, as well as fewer adverse effects or drug interactions [20,21].

Many women with epilepsy need long-term AED therapy. Approximately $70 \%$ of female patients are free from seizure with monotherapy $[22,23]$. The remaining $30 \%$ of women require multiple AEDs for effective seizure control [24]. Many of these AEDs are prescribed without consideration of the potential impact on or of concomitant hormone contraceptive use.

\section{Hormonal contraceptives for women with epilepsy}

Contraceptive management in women with epilepsy is critical owing to potential maternal and fetal risks $[16,22,25]$. Therefore, treatment guidelines for women with epilepsy need to include recommendations on contraception. There are two distinct categories of birth-control options for women: hormonal and non-hormonal methods. Nonhormonal methods include intrauterine devices (IUDs), barrier methods, such as condoms, diaphragms and cervical caps, as well as the rhythm method. A wide range of hormonal methods of contraception are available (Box 1) [26]. Long-acting hormonal contraceptives include injectable progestogens, subdermal implants and hormone-releasing IUDs. The efficacy of agents such as oral contraceptives (OCs), also known as combined OC pills, and contraceptive patches, is highly dependent on correct use and individual lifestyles. Unlike barrier methods, hormonal contraceptives do not protect against HIV infection and other sexually transmitted diseases. The rhythm method or other methods that depend on hormonal changes are not reliable methods of birth control.

The OCs are among the most widely used agents, since these preparations are highly effective when used properly. Designed to simulate the 28 days of the natural menstrual cycle, most OCs consist of an estrogenic and/or a progestogenic agent. A variety of OCs are available, with substantially different components, doses and side effects (Table 2). The primary mechanism underlying OC action is inhibition of ovulation. Although they essentially act by suppression of gonadotropins by feedback actions of estrogenic and/or progestogenic components, other effects include changes in cervical mucus, and the endometrium. Two types of OC pills are widely available: combination pills and progestogen-only pills (Table 2). The combined daily OC pill is composed of low-dose synthetic estrogen and progestogen. They are usually taken for 21 days with a 7-day gap (usually filled with either sugar or iron pills), during which withdrawal bleeding occurs. The two major synthetic estrogens used in OCs are ethinyl estradiol and mestranol. Mestranol is a prodrug and, hence, is inactive until it is converted to ethinyl estradiol in the body (mestranol is not available in the USA). The main synthetic progestogens include norethindrone, levonorgestrel, norgestimate, norgestrel, desogestrel and drospirenone.

Currently available combined OCs can be divided into three types: monophasic (only one dose of estrogen and progestogen during the 21 days), biphasic (varying doses of estrogen and progestogen) and triphasic (varying doses of estrogen and progestogen) (Table 2). In monophasic combinations, the progestogen and estrogen are present in fixed amounts and, hence, the blood levels rise and fall together. Biphasic and triphasic combinations are developed to mimic nearly physiological levels. In the biphasic regimen, the progestogen dose is increased during the last 11 days of the cycle, while the triphasic regimen consists of a progestogen/estrogen regimen that is changed three times during the cycle by altering the doses of either progestogen or estrogen or both. The progestogen-only pill - 'minipill' - contains progestogen without estrogen and is taken continuously. There are two progestogen-only OCs: 
Micronor and Ovrette (Table 2). However, they are slightly less frequently used than combination OCs.

Several nonconventional OC preparations, such as Seasonale ${ }^{\circledR}$ (consisting of 84 tablets containing levonorgestrel and ethinyl estradiol, followed by 7 days of placebo pills) and Lybrel $^{\circledR}$ (a continuous-use, low-dose OC containing levonorgestrel $90 \mu \mathrm{g}$ and ethinyl estradiol $20 \mu \mathrm{g}$ in a 28-day pack with all active tablets), are available for limiting menstrual intervals or eliminating menstrual periods [27]. Seasonique ${ }^{\circledR}$ consists of 84 pills each containing levonorgestrel $0.15 \mathrm{mg}$ and ethinyl estradiol $0.03 \mathrm{mg}$, and seven pills containing ethinyl estradiol $0.01 \mathrm{mg}$. These preparations are usually referred to as extended-cycle regimens. Nonoral hormonal contraceptive preparations include transdermal patches (Ortho Evra ${ }^{\circledR}$ ), vaginal rings (NuvaRing ${ }^{\circledR}$ ), subcutaneous implants and IUDs containing progesterone, and progestogen injections administered every 3 months (Depo Provera ${ }^{\circledR}$ ). NuvaRing is a once-amonth vaginal contraceptive that releases a continuous low dose of estrogen and progestin. The Ortho Evra patch provides monophasic combination of norelgestromin $(6 \mathrm{mg})$ and ethinyl estradiol $(0.75 \mathrm{mg}$ ) delivered via a transdermal system (one patch per week for 3 weeks and then no patch for 1 week). Combined and progestogen-only pills are taken in high doses for emergency contraception (EC) - referred as the morning-after pill or postcoital pills. There are two preparations for postcoital EC: Plan-B ${ }^{\circledR}$ (levonorgestrel) and Preven ${ }^{\mathrm{TM}}$ (levonorgestrel plus ethinyl estradiol). These EC products are effective ( $90 \%)$ if taken within $72 \mathrm{~h}$ after coitus.

\section{Box 1. Overview of various types of hormonal contraceptive agents}

\section{Oral contraceptives}

- Combined oral contraceptive pill (see Table 2)

- Progesterone-only pill (see Table 2)

\section{Nonsurgical preparations}

- Contraceptive patch (Ortho Evra $\left.{ }^{\circledR}\right)$

- Contraceptive vaginal ring $\left(\mathrm{NuvaRing}^{\circledR}\right)$

\section{Surgical devices/preparations}

- Progesterone implants

- Progesterone intrauterine devices

\section{Injection preparations}

- $\quad$ Progestogen injection (Depo Provera ${ }^{\circledR}$ )

\section{Use of hormonal contraceptives \& seizure risk}

There is no strong evidence that the use of OCs increase the risk of seizures in many women with epilepsy. Although some reports suggest that OCs might exacerbate seizures, most studies show no effect of estrogen-based contraceptives on seizure frequency [28,29]. A large cohort study showed that OCs do not increase the incidence of epilepsy [30]. By contrast, OC administration may alleviate the menstrually related seizures. Provera ${ }^{\circledR}$ (medroxyprogesterone acetate) was reported as a $3 \alpha$-hydroxysteroidoxidoreductase inhibitor, which implies that this contraceptive agent might enhance synthesis of inhibitory neurosteroids in the brain [31]. Drospirenone, a progestin component of OCs, has antiandrogenic effects. This agent is currently used for the treatment of hyperandrogenism characterizing polycystic ovaries. For women with epilepsy whose seizures are influenced by low premenstrual progesterone levels, progesterone or a synthetic progestogen supplementation during days 10-26 of the menstrual 
cycle is recommended [12]. Herzog studied progesterone as adjunctive antiepileptic therapy on women with intractable localization-related epilepsy or women with catamenial seizure exacerbation and reported a significant decrease in seizure frequency [12]. The synthetic 17 $\alpha$-ethynyl estradiol is a cytochrome P450 (CYP)3A4 inhibitor [32]. As seen in the following section, CYP3A4 is the primary enzyme metabolizing progestogens.

There is serious concern regarding the effect of hormonal contraceptives on the risk of seizures in women with epilepsy. However, there is controversy regarding the effects of OCs on seizures in women with epilepsy. Some women experienced seizure exacerbations with OCs, but clinical evidence is very limited. Recently, Herzog and colleagues from the Progesterone Trial Group have reported results of a pilot data that show seizure exacerbation with hormonal contraception [33]. Approximately $23 \%$ of patients (31 out of 141 women with epilepsy) reported seizure exacerbation in relation to past hormonal contraception. There was no significant difference in the proportion of women with seizure worsening between the catamenial and noncatamenial epilepsy groups. Future research is certainly warranted to further establish the effect of OCs on seizures in women with epilepsy.

\section{Pharmacokinetic interactions: effect of enzyme-inducing AEDs on OCs}

Many AEDs can influence the performance of hormonal contraceptives, which poses a serious risk owing to potential AED-induced teratogenicity [29,34]. Studies indicate that OC failures are the cause of one in four unplanned pregnancies in women with epilepsy [35]. OCs, when used properly, have an annual failure rate of $1 \%$ in healthy women. Based on a population study in 82 women with epilepsy by Coulam and Annegers in 1979, the annual failure rate derived from the 41 women who took OCs and AEDs together was approximately 6\% [34]. In another study, the failure rate for OCs in women with epilepsy was $3 \%$ compared with less than $1 \%$ in the general population [36]. Therefore, practice guidelines recommend highly effective contraception for women with epilepsy, since these women have a higher risk of pregnancy than healthy women.

Estrogens and progestogens are both susceptible to drug interactions with AEDs. CYP is a group of enzymes that metabolize drugs to a more water-soluble form, rendering them available for renal excretion. AEDs are categorized as enzyme-inducing and nonenzyme-inducing types (Box 2). The majority of AEDs are eliminated via hepatic CYP isoenzymes, especially CYP3A4 (Figure 1).

Antiepileptic drugs, such as phenobarbital, carbamazepine, phenytoin, felbamate, topiramate, primidone and oxcarbazepine, are mainly metabolized by the CYP3A4 enzyme system [37], which is also the primary enzyme metabolizing estrogens and progestogens (Figure 1). These AEDs can increase the activity of CYP3A4. Induction of the 3A4 system increases the metabolism of both the estrogenic and progestogenic components of OCs and reduces their circulating levels by as much as 50\% [34,38]. This could lead to reduced efficacy of all hormonal contraceptives, such as the low-dose daily-intake pills and low-releasing subdermal progestogen implants $[39,40]$. Therefore, enzyme-inducing AEDs can decrease serum levels of the OC steroids, leading to increased risk of contraception failure. In this regard, phenobarbital, carbamazepine and phenytoin are more potent CYP3A4 inducers than other enzyme-inducing AEDs (Box 2). Phenytoin can significantly decrease the AUC of both ethinyl estradiol and levonorgestrel (by 50\%) [37,38]. Similarly, carbamazepine can markedly reduce levels of ethinyl estradiol when taken concurrently (by 66\%) [38].

In addition, phenobarbital, phenytoin and carbamazepine have been shown to increase levels of SHBG, which decrease the unbound and, hence, the biologically active portion of the circulating estrogen and progestogen [41]. Sex hormones, such as progestogens, are highly bound to SHBG and albumin ( $>95 \%)$. Women treated with carbamazepine showed significant 
increase in serum levels of estradiol:SHBG ratio [42]. Hence, the SHBG-related alterations in distribution of OCs could also partly contribute to OC failure.

Several newer AEDs - felbamate, topiramate and oxcarbazepine - while being less-potent inducers of CYP3A4 [20,21], have been shown to accelerate the metabolism of estrogenic and progestogenic components of OCs [40]. Oxcarbazepine (1200 mg/day) was studied in healthy women volunteers who were taking a combination OC consisting of ethinyl estradiol and levonorgestrel [43]. Oxcarbazepine decreased the AUC of both components by 47\%. The interaction between felbamate and combination OCs was evaluated in healthy women in a randomized, double-blind, placebo-controlled study [44]. The enzyme-inducing effect of both carbamazepine and oxcarbazepine on CYP3A4 was investigated recently [45]. Oxcarbazepine was significantly less efficient as an enzyme inducer than carbamazepine. Oxcarbazepine is primarily converted to active monohydroxy derivatives via reduction and conjugation. Only a very small portion $(\sim 4 \%)$ is directly oxidized into the inactive dihydroxy derivative via CYP450 isoenzymes [46]. Topiramate reduced the AUC of the estrogenic component ethinyl estradiol by $18-30 \%$ in a dose-related fashion [47-49]. However, there was no effect of topiramate on the progestogen norethindrone at all doses tested. Compared with carbamazepine, topiramate demonstrated a much lower impact on metabolism of combined OC (Ortho-Novum ${ }^{\circledR}$ ) containing norethindrone and ethinyl estradiol. However, high doses of oxcarbazepine and topiramate may have significant effects on contraceptive steroids diminution. Topiramate (>200 mg/day) was reported to increase the oral clearance of ethinyl estradiol [50]. Felbamate treatment resulted in a significant $42 \%$ decrease in the progestogen component gestodene AUC, whereas no such effect was observed on the pharmacokinetic parameters of ethinyl estradiol. Despite felbamate's reduction of gestodene's AUC, there was no evidence of contraception failure.

The American Academy of Neurology, the American Epilepsy Society and the International League against Epilepsy have issued guidelines for the pharmacotherapy of epilepsy. Specific guidelines on the choice of contraception and drug interactions in women with epilepsy have never been published. In the article on the initial management of epilepsy by French and Pedley in 2008 in the New England Journal of Medicine, the authors made the following recommendations [51]:

- Women with epilepsy who are treated with enzyme-inducing AEDs, such as phenytoin, carbamazepine or topiramate, should receive preparations with a higher content of ethinyl estradiol;

- Start with an OC pill with estrogen $50 \mu \mathrm{g}$ at least and, if breakthrough bleeding occurs, an even higher estrogen dose may be needed.

Box 2. Antiepileptic drugs and their interactions with oral contraceptives

Antiepileptic drugs that may interact with oral contraceptives (enzyme-inducing antiepileptic drugs)

- Carbamazepine $\left(\right.$ Tegretol $\left.^{\circledR}\right)$

- Felbamate (Felbatol $\left.{ }^{\circledR}\right)$

- $\quad$ Lamotrigine $\left(\text { Lamictal }{ }^{\circledR}\right)^{\dagger}$

- Oxcarbazepin (Trileptal ${ }^{\circledR}$ )

- Phenobarbitone (Luminal ${ }^{\circledR}$ )

- Phenytoin (Dilantin ${ }^{\circledR}$ )

- Primidone (Mysoline ${ }^{\circledR}$ ) 
- $\quad$ Topiramate $\left(\right.$ Topamax $\left.^{\circledR}\right)$

Antiepileptic drugs that do not interact with oral contraceptives (enzyme-noninducing antiepileptic drugs)

- Clonazepam $\left(\right.$ Rivotril $\left.^{\circledR}\right)$

- Ethosuximide (Zarontin ${ }^{\circledR}$ )

- Gabapentin (Neurontin ${ }^{\circledR}$ )

- Levetiracetam (Keppra $\left.{ }^{\circledR}\right)$

- Pregabalin $\left(\right.$ Lyrica $\left.^{\circledR}\right)$

- Tiagabine (Gabitril ${ }^{\circledR}$ )

- Valproate (Depakote ${ }^{\circledR}$ )

- Vigabatrin $\left(\right.$ Sabril $\left.^{\circledR}\right)$

- Zonisamide (Zonegran ${ }^{\circledR}$ )

†'Weak enzyme inducer.

Despite these changes in OC regimens, the failure rate may still be higher than normal. Therefore, consideration must be given to an additional method of contraception. It is unclear how much of a decrease in either the estrogenic or progestogenic levels by enzyme-inducing AEDs will permit pregnancy to occur, making it difficult to estimate how the OC regimen should be optimized to restore maximal efficacy. Obviously, it is reasonable to consider changing to noninducing AEDs if the spectrum of AEDs fits the seizure type. The dosage of lamotrigine requires adjustment when OCs are started or discontinued because OCs can enhance the clearance of lamotrigine. Nonhormonal, highly effective methods, such as IUDs, may be ideal for women with epilepsy, since the contraceptive mechanism of IUDs is unaffected by changes in hepatic enzyme activity. The use of IUDs would alleviate druginteraction problems with AEDs.

\section{Pharmacokinetic interactions: effect of enzyme noninducing AEDs on OCs}

Antiepileptic drugs that do not induce CYP3A4 (i.e., valproate, gabapentin, levetiracetam, tiagabine, vigabatrin, zonisamide and pregabalin) belong to the second generation of AEDs and are less likely to interact with OCs. Consequently, the second-generation AEDs have less impact on hormonal contraception than many first-generation AEDs [40]. Hence, treatment with these AEDs in women taking OCs is relatively straightforward compared with enzymeinducing AED. Although valproate does not induce CYP3A4, it may be associated with a risk of endocrine dysfunction in women, such as increased incidence of polycystic ovarian disease [29]. Zonisamide showed stable plasma concentrations of the components ethinyl estradiol and norethindrone when it was coadministrated with a combined OC [52]. Levetiracetam was reported not to interact with low-dose contraceptive pills [53]. The 500-mg twice-daily dosage of levetiracetam administration on healthy females did not show any impact on the plasma levels of OCs. However, there is growing evidence of an interaction between lamotrigine and OCs that results in lower levels of lamotrigine, as described later.

Other hormonal contraception methods, such as medroxyprogesterone acetate (Depo Provera) depot injection and implantable hormonal contraceptive (Norplant ${ }^{\circledR}$ ) or hormone-releasing IUDs, may have some interaction with AEDs [41]. Norplant is a contraceptive system consisting of levonorgestrel (progestogen) formulated within small, silastic-covered rods. These rods are placed under the skin of the inside of the lower arm. The progestogen is slowly 
released and the system protects against pregnancy for up to 3 years. Norplant has been associated with unplanned pregnancies in women taking enzyme-inducing AEDs [54]. However, Norplant is no longer available on the US market. Currently, Implanon ${ }^{\circledR}$, another low-dose progesterone implant, is available. This progestogen-only single rod, introduced under the skin, protects for 3 years but is contraindicated with enzyme-inducing AEDs. The high-dose, injectable, progestin-only formulations are highly effective contraceptives in general female population. Depo Provera injections are contraceptive injections, which may be considered by women with epilepsy. As with combined OCs, the efficacy of Depo Provera may be reduced if taken with enzyme-inducing AEDs (Box 2). This limitation with Depo Provera can be partly overcome by changes in injection regimen, such as repeating injections every 10 weeks rather than the usual 12 weeks, especially when taking enzyme-inducing AEDs. However, there is no consensus on this recommendation owing to lack of studies directly evaluating the effectiveness of Depo Provera in women with epilepsy. With the transdermal patch, Ortho Evra, there are few trials that have been undertaken in women taking enzymeinducing AEDs. There is no evidence of interaction of AEDs with nonhormonal contraception methods, such as copper-containing IUDs or any barrier method [16,25]. The classic IUD or the newer levonorgestrel-releasing IUDs are an alternative for women with epilepsy, especially if they are taking enzyme-inducing AEDs or lamotrigine.

\section{Pharmacokinetic interactions: effect of OCs on AEDs}

There are several reports of pharmacokinetic interactions of OCs with AEDs, which raise concern regarding increased risk of seizures. Some OCs decrease serum AED concentration by increasing AED metabolism [55]. Galimberti et al. found that apparent clearance of total and unbound serum valproic acid concentrations were increased in women taking combined hormonal contraceptive steroids [56]. Lamotrigine levels were significantly reduced by OCs $[55,57]$. Since the major route of elimination of lamotrigine is by conjugation with glucuronic acid, and ethinyl estradiol of the combined OC is a well-known inducer of uridine-diphosphate glucuronsyl transferase isoenzymes [58], the higher clearance rate of lamotrigine was expected. Progestogen-only pills do not alter lamotrigine serum concentrations [59]. The study by Christensen et al. indicated that uridine-diphosphate glucuronsyl transferase metabolism of lamotrigine was increased by a common combined oral contraceptive administration (ethinyl estradiol $35 \mu \mathrm{g} /$ norgestimate $250 \mu \mathrm{g}$ ) [60]. Sidhu et al. in the reported a significant decrease of lamotrigine $\mathrm{AUC}_{24-\mathrm{h}}$ combined OC treatment [61]. The apparent lamotrigine clearance was increased 2.1-fold. It was also reported that combined OCs reduced the blood level of lamotrigine by $40-60 \%$ if the patient was not already taking enzyme-inducing AEDs. Thus, this interaction has major implications in seizure control in women. When women taking lamotrigine also begin OC use, the level of lamotrigine may decline by $50 \%$ and increase seizure risk [25]. To prevent this interaction, lamotrigine levels should be checked before and after starting OCs, and dose adjustments should be made based on clinical monitoring for seizure control and lamotrigine levels.

\section{Expert commentary}

Combination OCs are prescribed for approximately $17 \%$ of women with epilepsy at childbearing age, while $25 \%$ women in the general population use OCs. Women with epilepsy at childbearing age face unique issues regarding contraception. Contraceptive management in women with epilepsy is critical owing to potential maternal and fetal risks if either contraception or seizure management fails. Women with epilepsy have many options for birth control, including hormonal and nonhormonal agents. Combination OCs are most widely used in fertile women with epilepsy. Additional consideration should be given to the effect of OCs on seizures and also the potential drug interactions with concurrent drugs that may diminish the effectiveness of OCs. The most frequently asked question is whether OCs per se affect 
seizure susceptibility. There is controversy regarding the effects of OCs on seizures in women with epilepsy. Women experienced seizure exacerbations with OCs, but clinical evidence is very limited. Most studies show no effect of estrogen-based contraceptives on seizure frequency. Future research is certainly warranted for further defining the effect of OCs on seizures in women with epilepsy.

There is significant evidence of interactions between AEDs and OCs that can influence drug efficacy. AEDs provide satisfactory seizure control in women with epilepsy but a greater proportion of women show resistance or inadequate seizure control with current AEDs. Moreover, the enzyme-inducing AEDs (phenytoin, phenobarbital, carbamazepine, felbamate, topiramate, oxcarbazepine and primidone) can interact with OCs and, thereby, reduce the efficacy of hormonal contraception. This is thought to result from the induction of CYP3A4 metabolism of estrogen/progestogens and also from increased synthesis of SHBGs. This reduced efficacy is evident with all forms of hormonal contraceptives. The contraceptive failure is frequently attributed to the reduced concentrations of estrogenic component of the OCs. Estrogens are metabolized by hydroxylation to inactive metabolites by the CYP3A4 or by direct conjugation. AEDs that induce the CYP3A4 systems (carbamazepine, oxcarbazepine, phenobarbital, phenytoin and topiramate) accelerate the metabolism of estrogenic component of OCs and, thereby, reduce their efficacy. To partly overcome this limitation with enzymeinducing AEDs, formulations of OCs should contain at least $50 \mu \mathrm{g}$ of estrogen and or supplementary or alternative methods should be considered, such as the classical IUDs or an additional barrier method. The effectiveness of progesterone-only pills is also reduced if used in combination with enzyme-inducing AEDs.

Importantly, AEDs not interacting with OCs are a preferable treatment choice for women with epilepsy. The newer AEDs (gabapentin, levetiracetam, tiagabine and zonisamide) that do not induce hepatic enzymes should be considered. OCs can increase the metabolism of some AEDs such as lamotrigine and, thereby, reduce its effective concentrations [55,62]. This may have serious consequences, including increasing the risk of seizures. Increased metabolism of lamotrigine by OC induction of the uridine diphosphate glucuronosyltransferase system has been demonstrated as the likely mechanism. Subdermal implants are not recommended in women using enzyme-inducing AEDs owing to their high failure rate. Nonhormonal methods of contraception, such as copper IUDs or barrier methods, can be considered as alternate methods. Despite extensive information regarding the drug-drug interactions with different OCs, there is a large in gap in knowledge of interactions between OCs and AEDs. The newer AEDs have not been investigated extensively. Further studies are clearly warranted to address critical issues, such as optimization of AED therapy for seizure control while achieving effective contraception with OCs. The majority of women with epilepsy are not completely aware of drug interactions of OCs with AEDs. Patient-awareness programs on drug interactions with OCs may improve contraception efficacy. Future research directions include further defining the effects of OCs on seizures in women with epilepsy, and evaluation of novel therapeutic approaches, such as neurosteroids for women with epilepsy.

\section{Five-year view}

As discussed previously, contraceptive management in women with epilepsy is an important issue to avoid serious risks of contraception failure and fetal injury by some AEDs. Concurrent therapy of certain AEDs with OCs can result in reduced efficacy of OCs owing to AED induction of CYP3A4 metabolism of estrogen/progestogens. There is much effort to prevent this drug interaction in women with epilepsy. In 5 years, improved educational projects for patients and physicians will be developed to highlight the potential drug interactions between OCs and AEDs. Development of professional guidelines for the management of epilepsy in women will be developed in the next few years. The controversial issue that is being actively 
investigated is whether OCs exacerbate seizures in women with epilepsy. Although pilot studies do support the notion that some women may experience increased seizures with OCs, a large study is warranted to further characterize the effect of hormonal contraceptives on seizure frequency in women with epilepsy. Additional pharmacokinetic studies with newer AEDs are essential to establish their effects on OCs and also the effect of OCs on newer AEDs. In the next few years, the results of a progesterone trial will define the therapeutic utility of progesterone and related neurosteroids in women with epilepsy.

\section{Key issues}

- Women at childbearing age with epilepsy face unique issues regarding contraception.

- Contraceptive management in women with epilepsy is critical owing to the potential maternal and fetal risks if contraception or seizure management fails.

- The effect of oral contraceptives (OCs) on seizures is controversial. In women with epilepsy, there is no strong evidence of seizures worsening with OC use.

- Pharmacokinetic factors play a key role in the choice of antiepileptic drug (AED) therapy and hormonal contraception, since enzyme-inducing AEDs can reduce the efficacy of OCs owing to drug interactions.

- Estrogens and progestogens are metabolized by cytochrome P450 3A4 systems, which are generated by enzyme-inducing AEDs, such as phenytoin, phenobarbital, carbamazepine, topiramate and oxcarbazepine.

- Moreover, OCs can reduce effective concentrations of lamotrigine and, thereby, increase the risk of seizures.

- Newer AEDs that do not interact with OCs can be considered for women using OCs.

- Nonhormonal strategies, such as intrauterine devices or barrier methods, should be recommended as alternate methods of contraception in women taking AEDs.

- Patient-awareness programs on AED interactions with OCs may improve contraception efficacy in women with epilepsy.

- Professional guidelines should be developed for the improved management of epilepsy in women.

\section{Acknowledgments}

The author would like to thank Wendy Zhang for helpful discussion.

\section{References}

Papers of special note have been highlighted as:

- of interest

- of considerable interest

1. El-Sayed YY. Obstetric and gynecologic care of women with epilepsy. Epilepsia 1998;39(Suppl. 8):S17-S25. [PubMed: 9915616]

2. Harden CL, Pennell PB, Koppel BS, et al. Management issues for women with epilepsy - focus on pregnancy (an evidence-based review): III. Vitamin K, folic acid, blood levels, and breast-feeding: Report of the Quality Standards Subcommittee and Therapeutics and Technology Assessment 
Subcommittee of the American Academy of Neurology and the American Epilepsy Society. Epilepsia 2009;50:1247-1255. [PubMed: 19507305]

3. Morrell MJ. Reproductive and metabolic disorders in women with epilepsy. Epilepsia 2003;44(Suppl. 4):11-20. [PubMed: 12823565]

4. Pennell PB. 2005 AES annual course: evidence used to treat women with epilepsy. Epilepsia 2006;47 (Suppl. 1):46-53. [PubMed: 17044826]

5. Reddy DS. The role of neurosteroids in the pathophysiology and treatment of catamenial epilepsy. Epilepsy Res 2009;85:1-30. [PubMed: 19406620] • Comprehensive review on the role of endogenous neurosteroids in experimental animals and clinical catamenial seizures in women with epilepsy.

6. Hatcher, RA.; Nelson, A. Combined hormonal contraceptive methods. In: Hatcher, RA., editor. Contraceptive Technology. 18. Ardent Media; NY, USA: 2004. p. 391-460.

7. Sabers A. Pharmacokinetic interactions between antiepileptic drugs and oral contraceptives. Seizure 2008;17:141-144. [PubMed: 18206393]

8. Pack AM, Davis AR, Kritzerb J, Ava Yoonb A, Camus A. Antiepileptic drugs: are women aware of interactions with oral contraceptives and potential teratogenicity. Epielpsy Behav 2008;14:640-644.

9. Herzog AG, Klein P, Rand BJ. Three patterns of catamenial epilepsy. Epilepsia 1997;38:1082-1088. [PubMed: 9579954]

10. Herzog AG, Harden CL, Liporace J, et al. Frequency of catamenial seizure exacerbation in women with localization related epilepsy. Ann Neurol 2004;56:431-434. [PubMed: 15349872]

11. Bazan AC, Montenegro MA, Cendes F, Min LL, Guerreiro CA. Menstrual cycle worsening of epileptic seizures in women with symptomatic focal epilepsy. Arg Neuropsiguiatr 2005;63:751-756.

12. Herzog AG. Progesterone therapy in women with epilepsy: a 3-year follow-up. Neurology 1999;52:1917-1918. [PubMed: 10371551]

13. Reddy DS, Castaneda DC, O'Malley BW, Rogawski MA. Anticonvulsant activity of progesterone and neurosteroids in progesterone receptor knockout mice. J Pharm Exp Therap 2004;310:230-239.

14. Reddy DS, Kim HY, Rogawski MA. Neurosteroid withdrawal model of perimenstrual catamenial epilepsy. Epilepsia 2001;42:328-336. [PubMed: 11442149]

15. Luef G, Abraham I, Haslinger M, et al. Polycystic ovaries, obesity and insulin resistance in women with epilepsy. J Neurol 2002;249:835-841. [PubMed: 12140666]

16. Crawford P. Best practice guidelines for the management of women with epilepsy. Epilepsia 2005;46 (Suppl. 9):117-124. [PubMed: 16302885] - Insightful review on managing epilepsy in women of childbearing age.

17. Pimentel J. Current issues on epileptic women. Curr Pharma Design 2000;6:865-872.

18. Tatum WO, Liporace J, Benbadis SR, Kaplan PW. Updates on the treatment of epilepsy in women. Arch Intern Med 2004;164:137-145. [PubMed: 14744836]

19. Isojärvi JIT, Taubøll E, Herzog AG. Effect of antiepileptic drugs on reproductive endocrine function in individuals with epilepsy. CNS Drugs 2005;19:207-223. [PubMed: 15740176]

20. Rogawski MA, Löscher W. The neurobiology of antiepileptic drugs. Nat Rev Neurosci 2004;5:553564. [PubMed: 15208697] • Excellent review and mechanistic discussion of current available antiepileptic drugs.

21. Schmidt D. Drug treatment of epilepsy: options and limitations. Epilepsy Behav 2009;5:56-65. [PubMed: 19236951]

22. Leppik IE, Wolff D, Purves S. Treatment of epilepsy in women of childbearing potential. CNS Drugs 1999;11:191-206.

23. Sander JW. The use of antiepileptic drugs-principles and practice. Epilepsia 2004;45(Suppl. 6):2834. [PubMed: 15315513]

24. Patsalos PN. Clinical pharmacokinetics of levetiracetam. Clin Pharmacokinet 2004;43:707-724. [PubMed: 15301575]

25. O'Brien MD, Guillebaud J. Contraception for women with epilepsy. Epilepsia 2006;47:1419-1422. [PubMed: 16981856]

26. Reddy DS. Recent advances in hormonal contraceptives for women. Int J Pharma Sci Nanotech 2008;1:199-206. 
27. Anderson FD, Gibbons W, Portman D. Long-term safety of an extended-cycle oral contraceptive (Seasonale): a 2-year multicenter open-label extension trial. Am J Obstet Gynecol 2006;195:92-96. [PubMed: 16813747]

28. Burakgazi E, Harden C, Kelly JJ. Contraception for women with epilepsy. Rev Neurol 2009;6:E62E67.

29. Harden CL, Leppik I. Optimizing therapy of seizures in women who use oral contraceptives. Neurology 2006;67(Suppl. 4):S56-S58. [PubMed: 17190925] • Insightful review on the potential interactions between oral contraceptives OCs and antiepileptic drugs with expert clinical input for treatment optimization.

30. Vessey M, Painter R, Yeates D. Oral contraception and epilepsy. Contraception 2002;66:77-79. [PubMed: 12204778]

31. Belelli D, Herd MB. The contraceptive agent Provera enhances $\mathrm{GABA}_{\mathrm{A}}$ receptor-mediated inhibitory neurotransmission in the rat hippocampus: evidence for endogenous neurosteroids? J Neurosci 2003;23:10013-10020. [PubMed: 14602815]

32. Lin HL, Kent UM, Hollenberg PF. Mechanism-based inactivation of cytochrome P450 3A4 by 17 $\alpha-$ ethynylestradiol: evidence for heme destruction and covalent binding to protein. J Pharm Exp Ther 2002;301:160-167.

33. Herzog, AG.; Fowler, KM. Hormonal contraception and seizures. Presented at: American Epilepsy Society; WA, USA. 5-9 December 2008; Abstract 1.095

34. Coulam CB, Annegers JF. Do anticonvulsants reduce the efficacy of oral contraceptives? Epilepsia 1979;20:519-525. [PubMed: 477641]

35. Fairgrieve SD, Jackson M, Jonas P, et al. Population based, prospective study of the care of women with epilepsy in pregnancy. BMJ 2000;321:674-675. [PubMed: 10987772]

36. Kaneko S. Pregnancy and quality of life in women with epilepsy. Clin Therap 1998;20(Suppl. A):A30-A47. [PubMed: 9589812]

37. Crawford P. Interactions between antiepileptic drugs and hormonal contraception. CNS Drugs 2002;16:263-272. [PubMed: 11945109]

38. Crawford P, Chadwick DJ, Martin C, Tjia J, Back DJ, Orme M. The interaction of phenytoin and carbamazepine with combined oral contraceptive steroids. Br J Clin Pharmacol 1990;30:892-896. [PubMed: 2126946]

39. Penovich PE. The effects of epilepsy and its treatment on sexual and reproductive function. Epilepsia 2000;41(Suppl. 2):S53-S61. [PubMed: 10885740]

40. Perrucca E. Clinically relevant drug interactions with antiepileptic drugs. Br J Clin Pharmacol 2005;61:246-255.

41. Wilbur K, Ensom MHH. Pharmacokinetic drug interactions between oral contraceptives and secondgeneration anticonvulsants. Clin Pharmacokinet 2000;38:355-365. [PubMed: 10803456]

42. Lossius MI, Taubøll E, Mowinckel P, Mørkrid L, Gjerstad L. Reversible effects of antiepileptic drugs on reproductive endocrine function in men and women with epilepsy - a prospective randomized double-blind withdrawal study. Epilepsia 2007;48:1875-1882. [PubMed: 17555526]

43. Fattore C, Cipolla G, Gatti G, et al. Induction of ethinylestradiol and levonorgestrel metabolism by oxcarbazepine in healthy women. Epilepsia 1999;40:783-787. [PubMed: 10368079]

44. Saano V, Glue P, Banfield CR, et al. Effects of felbamate on the pharmacokinetics of a low-dose combination oral contraceptive. Clin Pharmacol Ther 1995;58:523-531. [PubMed: 7586946]

45. Andreasen AH, Brøsen K, Damkier P. A comparative pharmacokinetic study in healthy volunteers of the effect of carbamazepine and oxcarbazepine on CYP3A4. Epilepsia 2007;48:490-496. [PubMed: 17346248]

46. Schmidt D, Sachdeo R. Oxcarbazepine for treatment of partial epilepsy: a review and recommendations for clinical use. Epilepsy Behav 2000;1:396-405. [PubMed: 12737829]

47. Rosenfeld WE, Doose DR, Walker SA, Nay RK. Effect of topiramate on the pharmacokinetics of an oral contraceptive containing norethindrone and ethinyl estradiol in patients with epilepsy. Epilepsia 1997;38:317-323. [PubMed: 9070594]

48. Doose DR, Wang SS, Padmanabhan M, Schwabe S, Jacobs D, Bialer M. Do new antiepileptic drugs interact with oral contraceptives? Epilepsia 2003;44:540-549. [PubMed: 12681003]

Expert Rev Clin Pharmacol. Author manuscript; available in PMC 2011 January 1. 
49. Doose DR, Wang SS, Padmanabhan M, Schwabe S, Jacobs D, Bialer M. Effect of topiramate or carbamazepine on the pharmacokinetics of an oral contraceptive containing norethindrone and ethinyl estradiol in healthy obese and nonobese female subjects. Epilepsia 2003;44:540-549. [PubMed: 12681003] • Elegant open-label study for two 28-day cycles that confirmed lack of significant interaction of topiramate with OC Ortho-Novum ${ }^{\circledR}$ compared with the carbamazepineOC combination therapy.

50. Bialer M, Doose DR, Murthy B, et al. Pharmacokinetic interactions of topiramate. Clin Pharmacokinet 2004;43:763-760. [PubMed: 15355124]

51. French JA, Pedly TA. Initial management of epilepsy. N Engl J Med 2008;359:166-176. [PubMed: 18614784] • Expert and authoritative review of various strategies and current pharmacotherapy of epilepsy, followed by a review of formal guidelines and clinical recommendations, including guidelines for management of epilepsy in women.

52. Baulac M. Introduction to zonisamide. Epilepsy Res 2006;68(Suppl):S3-S9. [PubMed: 16413170]

53. Ragueneau-Majlessi I, Levy RH, Janik F. Levetiracetam does not alter the pharmacokinetics of an oral contraceptive in healthy women. Epilepsia 2002;43:697-702. [PubMed: 12102671]

54. Schindlbeck C, Janni W, Friese K. Failure of implanon contraception in a patient taking carbamazepine for epilepsy. Arch Gynecol Obstet 2006;273:255-256. [PubMed: 16208481]

55. Sabers A, Öhman I, Christensen J, Tomson T. Oral contraceptives reduce lamotrigine plasma levels. Neurology 2003;61:570-571. [PubMed: 12939444] • First study to demonstrate that lamotrigine levels were reduced by $50 \%$ in women taking this drug in combination Ocs.

56. Galimberti CA, Mazzucchelli I, Arbasino C, Canevini MP, Fattore C, Perucca E. Increased apparent oral clearance of valproic acid during intake of combined contraceptive steroids in women with epilepsy. Epilepsia 2006;47:1569-1572. [PubMed: 16981874]

57. Sabers A, Gram L. Lamotrigine pharmacokinetics during anticonception and pregnancy. Lett Drug Design Discovery 2006;3:321-322.

58. Contin M, Albani F, Ambrosetto G, et al. Variation in lamotrigine plasma concentrations with hormonal contraceptive monthly cycles in patients with epilepsy. Epilepsia 2006;47:1573-1575. [PubMed: 16981875]

59. Reimers A, Helde G, Brodtkorb E. Ethinyl estradiol, not progestogens, reduces lamotrigine serum concentrations. Epilepsia 2005;46:1414-1417. [PubMed: 16146436]

60. Christensen J, Petrenaite V, Atterman J, et al. Oral contraceptives induce lamotrigine metabolism: evidence from a double-blind, placebo-controlled trial. Epilepsia 2007;48:484-489. [PubMed: 17346247]

61. Sidhu J, Job S, Singh S, Philipson R. The pharmacokinetic and pharmacodynamic consequences of the co-administration of lamotrigine and a combined oral contraceptive in healthy female subjects. Br J Clin Pharmacol 2005;61:191-199. [PubMed: 16433873]

62. Herzog AG, Blum AS, Farina EL, et al. Valproate and lamotrigine level variation with menstrual cycle phase and oral contraceptive use. Neurology 2009;72:911-914. [PubMed: 19273825]

Expert Rev Clin Pharmacol. Author manuscript; available in PMC 2011 January 1. 


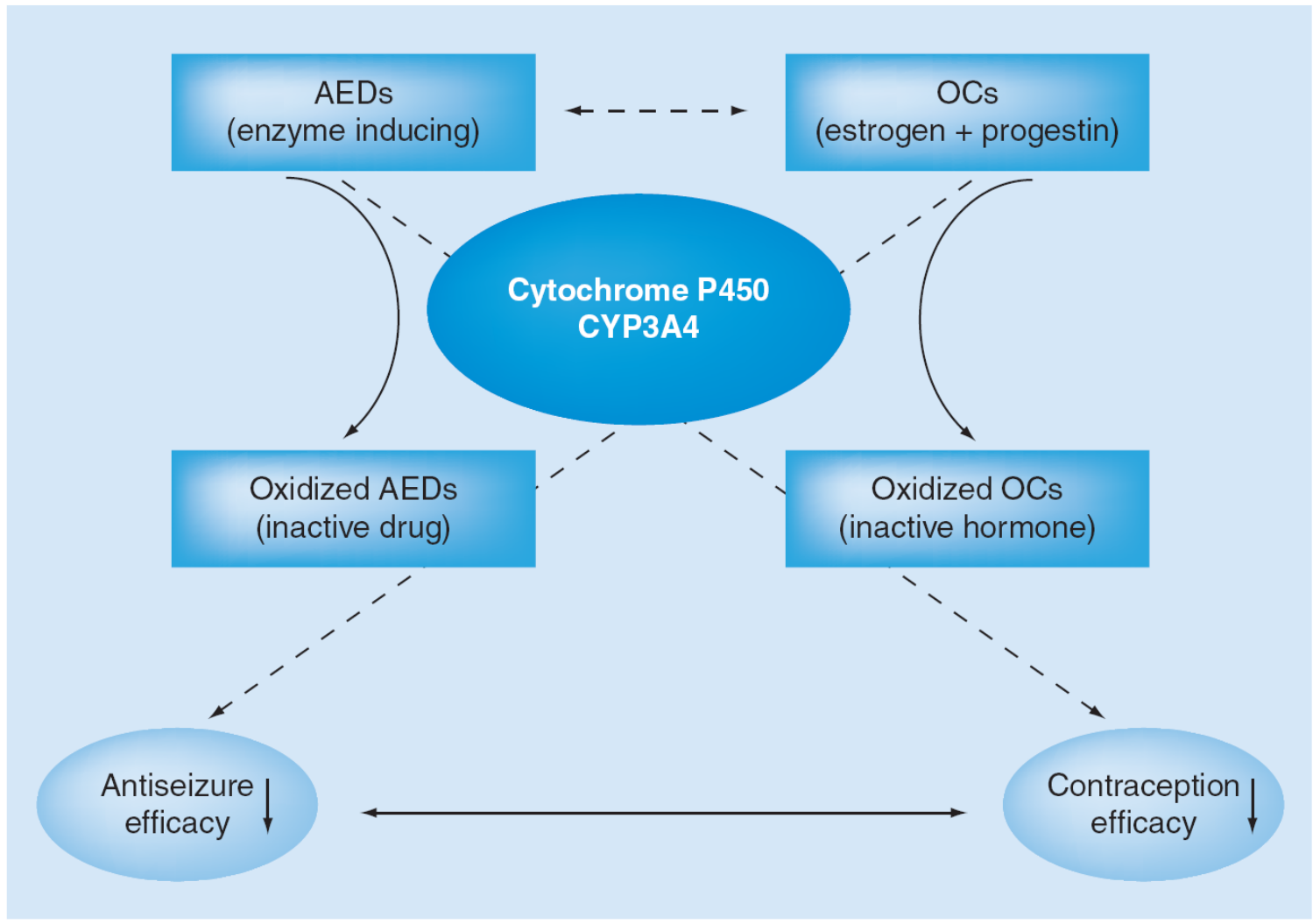

Figure 1.

Metabolism of antiepileptic drugs and the components of oral contraceptives by the common hepatic cytochrome P450 enzyme system, which can lead to pharmacokinetic interactions and, thereby, reduced therapeutic efficacy when taken together.

AED: Antiepileptic drug; OC: Oral contraceptive. 
Table 1

Overview of US FDA-approved antiepileptic drugs.

\begin{tabular}{lll}
\hline Antiepileptic drug & Year approved & Therapeutic efficacy \\
\hline First generation (standard) & \\
\hline Carbamazepine & 1974 & Broad spectrum \\
\hline Clonazepam & 1997 & Broad spectrum, status epilepticus \\
\hline Ethotoin & 1957 & Partial/generalized \\
\hline Methsuximide & $1982^{\dagger}$ & Absence seizures \\
\hline Phenobarbital & $1982^{\dagger}$ & Partial/generalized \\
\hline Phenytoin & $1982^{\dagger}$ & Partial/generalized \\
\hline Primidone & 1974 & Partial/generalized \\
\hline Valproic acid & 1983 & Broad spectrum \\
\hline Second generation $($ newer) & \\
\hline Acetazolamide & 1990 & Partial/generalized \\
\hline Ethosuximide & 2000 & Absence seizures \\
\hline Felbamate & 1993 & Broad spectrum, Lennox-Gastaut syndrome \\
\hline Fosphenytoin & 1996 & Status epilepticus \\
\hline Gabapentin & 2000 & Partial (add-on) \\
\hline Lacosamide & 2008 & Partial (add-on) \\
\hline Lamotrigine & 1994 & Broad spectrum \\
\hline Levetiracetam & 1999 & Partial/generalized \\
\hline Oxcarbazepine & 2000 & Partial/generalized \\
\hline Pregabalin & 2005 & Partial (add-on) \\
\hline Rufinamide & 2008 & Lennox-Gastaut syndrome \\
\hline Tiagabine & 1997 & Partial (add-on) \\
\hline Topiramate & 1996 & Broad spectrum \\
\hline Aproved before & Infantile spasms \\
\hline
\end{tabular}


Table 2

Oral contraceptive preparations.

\begin{tabular}{|c|c|c|}
\hline Brand name (generics) & Progestogen (mg) & Estrogen (mg) \\
\hline \multicolumn{3}{|l|}{ Combination pills } \\
\hline \multicolumn{3}{|l|}{ Monophasic $^{\dagger}$} \\
\hline Alesse $^{\circledR}$ (lessina, lutera, aviane) & Levonorgestrel (0.1) & Ethinyl estradiol (0.02) \\
\hline Lo-Ovral $^{\circledR}$ (cryselle, low-ogestrel, velivet) & Norgestrel (0.3) & Ethinyl estradiol (0.03) \\
\hline Demulen $^{\circledR}$ (zovia, kelnor) & Ethynodiol diacetate (1) & Ethinyl estradiol (0.035) \\
\hline Desogen ${ }^{\circledR} /$ Ortho-Cept ${ }^{\circledR}$ (solia, apri, reclipsen) & Desogestrel (0.15) & Ethinyl estradiol (0.03) \\
\hline Loestrin ${ }^{\circledR} /$ Loestrin- $\mathrm{Fe}^{\circledR}$ (microgestin, junel) & Norethindrone acetate (1) & Ethinyl estradiol (0.02) \\
\hline Nordette $^{\circledR} /$ Leven $^{\circledR}$ (portia, levora) & Levonorgestrel (0.15) & Ethinyl estradiol (0.03) \\
\hline Ortho-Novum ${ }^{\circledR} /$ Medicon $^{\circledR}$ (nortel, neocon) & Norethindrone (1) & Ethinyl estradiol (0.035) \\
\hline Ortho-Cyclen ${ }^{\circledR}$ (mononessa, previfem, sprintec) & Norgestimate $(0.25)$ & Ethinyl estradiol (0.035) \\
\hline Ovcon- $35^{\circledR}$ (balziva, femcon, zenchent) & Norethindrone $(0.4)$ & Ethinyl estradiol (0.035) \\
\hline Ovral $^{\circledR}$ (ogestrel) & Norgestrel (0.5) & Ethinyl estradiol (0.05) \\
\hline Yasmin $^{\circledR}$ (ocella) & Drospirenone (3) & Ethinyl estradiol (0.03) \\
\hline $\mathrm{Yaz}^{\circledR}$ & Drospirenone (3) & Ethinyl estradiol (0.02) \\
\hline \multicolumn{3}{|l|}{$\overline{\text { Biphasic }}$} \\
\hline Mircette $^{\circledR \neq}$ (kariva) & Desogestrel $(0.15)$ & Ethinyl estradiol (0.02/0.01) \\
\hline \multicolumn{3}{|l|}{ Extended-cycle regimens } \\
\hline Seasonale ${ }^{\circledR} \oint$ (quasense, jolessa) & Levonorgestrol (0.15) & Ethinyl estradiol (0.05/0.01) \\
\hline Seasonique ${ }^{\circledR I I}$ & Levonorgestrol (0.15) & Ethinyl estradiol (0.03/0.01) \\
\hline \multicolumn{3}{|l|}{$\overline{\text { Triphasic }^{\#}}$} \\
\hline Ortho Tri-Cyclen ${ }^{\circledR}$ (trisprintec, tri-previfem, tri-nessa) & Norgestimate $(0.18 / 0.215 / 0.25)$ & Ethinyl estradiol $(0.035 / 0.035 / 0.035)$ \\
\hline Ortho-Novum ${ }^{\circledR}-7 / 7 / 7$ & Norethindrone $(0.5 / 0.75 / 1)$ & Ethinyl estradiol $(0.035 / 0.035 / 0.035)$ \\
\hline Tri-Levlen ${ }^{\circledR} /$ Triphasi $^{\circledR}$ (enpresse, trivora) & Levonorgestrel $(0.05 / 0.75 / 0.125)$ & Ethinyl estradiol $(0.03 / 0.04 / 0.03)$ \\
\hline Tri-Noriny ${ }^{\circledR}$ (arinelle) & Norethindrone $(0.5 / 1 / 0.5)$ & Ethinyl estradiol $(0.035 / 0.035 / 0.035)$ \\
\hline \multicolumn{3}{|l|}{ Progesterone-only pills } \\
\hline Micronor $^{\circledR} /$ Nor- $^{-} D^{\circledR}$ (camilla, jolivette, errin, Nora-BE) & Norethindrone $(0.35)$ & \\
\hline Ovrette $^{\circledR}$ & Norgestrel $(0.075)$ & \\
\hline
\end{tabular}

${ }^{\dagger}$ Fixed amounts of the estrogen and progestogen are present in each pill, which is taken daily for 21 days, followed by 7 days with placebo (sugar) pills or iron preparations.

Contains 21 pills of fixed amounts of estrogen (ethinyl estradiol $0.02 \mathrm{mg}$ ) and progestogen (desogestrel $0.15 \mathrm{mg}$ ) and two placebo pills (rather than the regular seven placebo pills), and five pills of low-dose estrogen (ethinyl estradiol $0.01 \mathrm{mg}$ ).

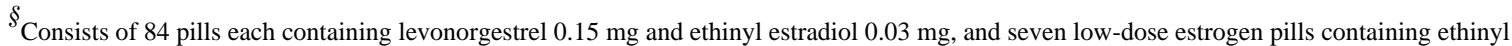
estradiol $0.01 \mathrm{mg}$.

${ }^{\text {II }}$ Consists of 84 pills each containing levonorgestrel $0.15 \mathrm{mg}$ and ethinyl estradiol $0.03 \mathrm{mg}$, followed by seven pills of placebo. This regimen is designed for one menstruation every 3 months. 
\# Provide three different pills containing varying amounts of estrogen and progestogen (7 days each regimen), to be taken at different times during the 21-day cycle. 